\title{
Diagnostic protocol for pancreatic neuroendocrine tumors (PNETs)
}

\author{
Modesto Varas ${ }^{1}$, Joan Gornals², José Luis Prieto ${ }^{3}$ and Julio Iglesias-García ${ }^{4}$. Grupo de trabajo de \\ Ultrasonografía Endoscópica de la SEPD
}

${ }^{1}$ Unit of Echoendoscopy. Centro Médico Teknon and Hospital Universitario del Valle Hebrón. Barcelona, Spain. ${ }^{2}$ Unit of Endoscopy. Hospital Universitario de Bellvitge. Hospitalet de Llobregat, Barcelona, and Centro Médico Teknon. Barcelona, Spain. ${ }^{3}$ Unit of Digestive Diseases. Hospital Punta Europa. Algeciras. Cádiz, Spain. ${ }^{4}$ Department of Digestive Diseases. Hospital Clínico Universitario de Santiago de Compostela. A Coruña, Spain

\begin{abstract}
ABBREVIATIONS
A: angiography; US: ultrasonography; EUS: endoscopic ultrasonography; IUS: intraoperative ultrasonography; EUS-FNA: endoscopic ultrasonography-guided fine-needle aspiration; MRI: magnetic resonance imaging; SRS: octreoscan; CT: computerized tomography; MDCT: multidetector computerized tomography; PET: positron emission tomography; PET-CT: PET with computerized tomography; ICC: immunocytochemistry; I: insulinoma; G: gastrinomas; PNET: pancreatic neuroendocrine tumor or apudoma; NF-PNET: non-functioning PNET; MEN: multiple endocrine neoplasia; VHL: von Hippel-Lindau disease; R: review; C: cystic; CE: contrast-enhanced.
\end{abstract}

\section{INTRODUCTION}

The advent of endoscopic ultrasonography (EUS) or echoendoscopy (EE) represented a breaking point in the localization and diagnosis of PNETs (1-4) (insulinomas, gastrinomas, glucagonomas, non-functioning, etc.) as it provided a high yield (sensitivity around 90\%, specificity at 98\%) (510 ) only second to EUS-FNA (almost 100\%) (10-15).

New EUS-related technologies such as contrast media and elastography have also improved PNET localization (16-22) with percentages matching those obtained with EUS-FNA.

A recent paper states that contrast agents (S: 95\%) substantially improve conventional EUS findings (21).

Received: 29-04-11.

Accepted: 01-09-11.

Correspondence: Modesto Varas. Unit of Echoendoscopy. Centro Médico Teknon. Marquesa de Vilallonga, 12. 08017 Barcelona. Spain.

e-mail: varas@dr.teknon.es
Varas M, Gornals J, Prieto JL, Iglesias-García J. Diagnostic protocol for pancreatic neuroendocrine tumors (PNETs). Rev Esp Enferm Dig 2012; 104: 29-32.

Therefore, diagnostic EUS should be now considered seriously for PNET assessment (10) in addition to elastography, contrast media, both things, or even FNA (22).

Furthermore, novel imaging techniques other than US (23), CT $(24,25)$, and MRI $(25,26)$, including PET (FDG \& DOPA) and PET-CT, may be used for the localization and staging of PNETs, particularly when no primary tumor has been found (27-32) (Table I).

When CT will not find a PNET, EUS does so in $91 \%$ of cases (34). According to several papers EUS is superior to MDCT (Multiple Detector Computerized Tomography) $(8,21,33,34)$.

PET-CT may be a match for Octreoscan (31) for tumors other than insulinomas, and only PET-CT is superior to Octreoscan when tumors with a high Ki-67 proliferation index are considered (32), with sensitivity approaching $100 \%$ when it comes to finding a primary tumor and its related metastases (35-38).

Once a tumor is precisely located its staging must ensue in order to decide on its appropriate management (surgical or otherwise) and to define a prognosis according to histopathology (Figs. 1 and 2) (39).

We have moved from the classical TNM system to the WHO histological classification (40):

- Well differentiated: benign, smaller than $2 \mathrm{~cm}$, confined to the pancreas, fewer than 2 mitoses per 10 HPFs, Ki-67 below 2\%, and chromogranin A +. No vascular invasion.

- Uncertain behavior: confined to the pancreas and one or more of the following: a) larger than $2 \mathrm{~cm}$; b) 2-10 mitoses; c) Ki-67 above 2\%; and d) vascular invasion and perineural permeation. 
Table I. A comparison of PNET detection rates with several imaging techniques

\begin{tabular}{|c|c|c|c|c|c|c|c|c|c|c|c|}
\hline Author & Year & $\mathrm{N} .^{\circ}$ & \% Insulinoma & Size & $\mathrm{CT}$ & EUS & MR & SRS & US & A & PET \\
\hline Zimmer & 1994 & 18 & & & & 88 & & 52 & & & \\
\hline Ueno & $1996(5)$ & 7 & $71 \%$ & & 57 & 100 & & & 86 & 100 & \\
\hline Proye & 1998 & 7 & & & & 77 & & & & & \\
\hline Anderson & $2000(6)$ & 54 & $58 \%$ & $15 \mathrm{~mm}$ & & 93 & & & & 44 & \\
\hline Thoeni & 2000 & & & & & & 80 & & & & \\
\hline Rickes & $2003(23)$ & 29 & & & & & & 54 & 94 US-CE & & \\
\hline Gouya & $2003(24)$ & 30 & $100 \%$ & $20 \mathrm{~mm}$ & 72 & 94 & & & & & \\
\hline Rappeport & 2006 (8) & 20 & $10 \%$ & $18 \mathrm{~mm}$ & 80 & 100 & & & & & \\
\hline Koopmans & 2008 (29) & 23 & & & 87 & & & 78 & & & 89 \\
\hline Alsohaibani & 2008 (9) & 14 & & $4-25 \mathrm{~mm}$ & 77 & $\begin{array}{l}100 \\
90 \text { USE-PAAF }\end{array}$ & 67 & 50 & & & \\
\hline Malagò & 2009 (42) & 38 & $0 \%$ & & & & & & 81.5 US-CE & & \\
\hline An & $2010(43)$ & 31 & $100 \%$ & & & & & & 89 US-CE & & \\
\hline Ishikawa & $2010(21)$ & 41 & & & 81 & 95 & & & 45 & & \\
\hline Suzuki & 2010 (15) & 34 & & $30 \mathrm{~mm}$ & 62 & $\begin{array}{l}65 \\
90 \text { USE-PAAF }\end{array}$ & & & 32 & & \\
\hline Druce & $2010(26)$ & 30 & $100 \%$ & & 64 & 65 & 75 & 50 & & & \\
\hline Versari & $2010(33)$ & 19 & & & 91 & 100 & & & & & 92 \\
\hline Khashab & $2011(34)$ & 60 & $32 \%$ & $32.7 \mathrm{~mm}$ & 63 & 92 & & & & & \\
\hline Gornals & $2011(14)$ & 9 y 16 casos & $33 \%$ & 19 mm & & 100 USEPAAF & & & & & \\
\hline Varas & 2011 (35) & 19 & $10 \%$ & $20 \mathrm{~mm}$ & 88 & 100 & & 80 & 83 & & 100 \\
\hline Turuga & 2011 (37) & Revisión & & & 80 & & 70 & 85 & & & 100 \\
\hline Tan & $2011(38)$ & Revisión & & & 94 & $80-90$ & & $80-90$ & $\begin{array}{l}<70 \text { sólo US } \\
66 \%\end{array}$ & & $90-100$ \\
\hline
\end{tabular}

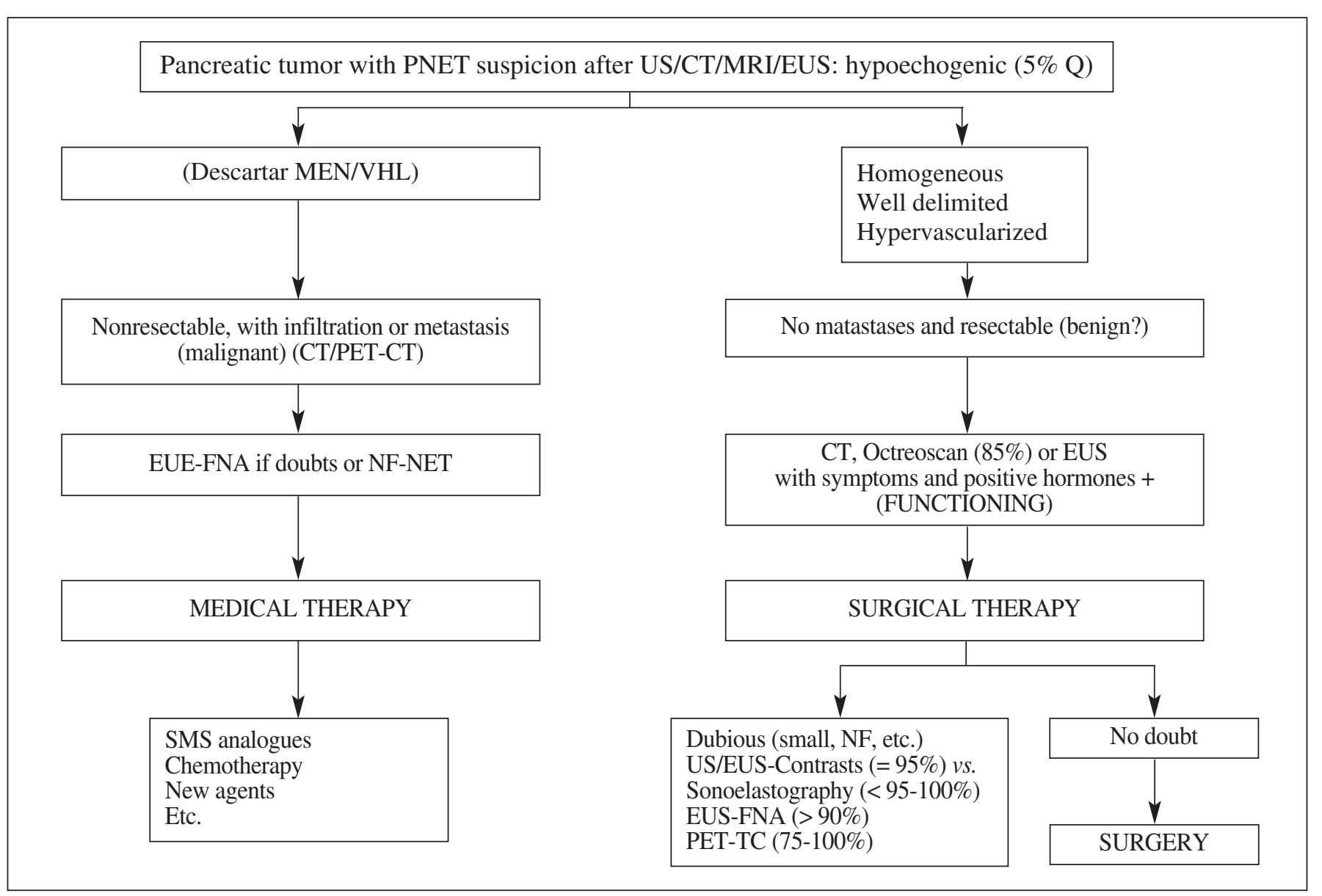

Fig. 1. General diagnostic algorithm (modified from reference 22). 


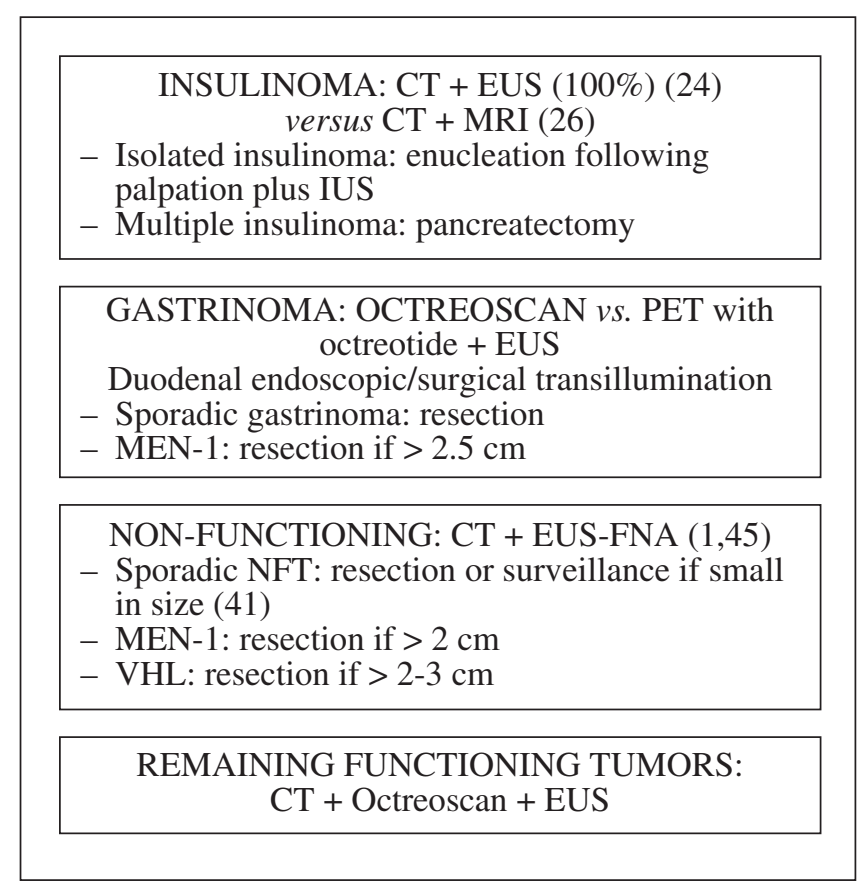

Fig. 2. Diagnostic algorithm for most common types.

- Well-differentiated endocrine carcinoma: low malignity. Macroscopic local invasion and/or metastasis (malignant). No vascular invasion.

- Poorly-differentiated endocrine carcinoma: high malignity, over 10-20 mitoses per 10 HPFs. Ki-67 above $15-20 \%$. Vascular invasion.

For instance, in a series of 139 NF-PNETs incidentally identified (mean size: $3 \mathrm{~cm}$ ) and then operated upon, $19 \%$ were classified as benign, $52 \%$ as with uncertain behavior, and $28 \%$ as malignancies. Mean 3-year follow-up of $80 \%$ (112 cases) revealed an actuarial survival of $89,92.5$, and $50 \%$, respectively, at 5 years (44).

\section{REFERENCES}

1. Alexakis N, Neoptolemos JP. Pancreatic neuroendocrine tumours. Best Pract Res Clin Gastroenterol 2008;22:183-205.

2. Varas MJ. Neuroendocrine tumors -fascination and infrequency. Rev Esp Enferm Dig 2009;101:195-208.

3. Diaz Roca AB, Iglesias Garcia J, Lariño-Noia J, Orive V, DomínguezMuñoz JE. Qué aporta la ultrasonografía endoscópica en el diagnóstico de los tumores neuroendocrinos del páncreas. Gastroenterol y Hepatol 2011;34:29-34

4. Kuiper P, Verspaget HW, Overbeek LIH, Biemond I, Lamers CB. An overview of the current diagnosis and recent developments in neuroendocrine tumours of the gastroenteropancreatic tract: the diagnostic approach. Netherlands J Med 2011;69:14-20.

5. Ueno N, Tomiyama T, Tano S, Wada S, Aizawa T, Kimura K. Utility of endoscopic ultrasonography with color doppler function for the diagnosis of islet cell tumor. Am J Gastroenterol 1996;91:772-6.

6. Anderson MA, Carpenter S, Thompson NW, Nostrant TT, Elta GH, Sheiman JM. Endoscopic ultrasound is highly accurate and directs managements in patients with neuroendocrine tumors of the pancreas. Am J Gastroenterol 2000; 95: 2271-7.
7. Varas MJ, Miquell JM, Maluenda MD, Boix J, Armengol-Miró JR. Preoperative detection of gastrointestinal neuroendocrine tumors using endoscopic ultrasonography. Rev Esp Enferm Dig 2006;98:828-36.

8. Rappeport ED, Hansen CP, Kjaer A, Knigge U. Multidetector computed tomography and neuroendocrine pancreaticoduodenal tumors. Acta Radiol 2006;47:248-56.

9. Alsohaibani F, Bigan D, Kneteman N, Shapiro AMJ, Sandha GS. The impact of preoperative endoscopic ultrasound on the surgical management of pancreatic neuroendocrine tumours. Can J Gastroenterol 2008;22:817-20.

10. Puli SR, Bechtold ML, Reddy JBk, Bapoje SR, Antillon MR, Brugge WR. Diagnostic accuracy of EUS in detecting pancreatic neuroendocrine tumors: a meta-analysis and systematic review. Gastroenterology 2009;136(5):Supl.1:A932.

11. Santo E, Kariv R, Monges G, Marmor S, Giovannini M. The role of linear array endoscopic ultrasound with fine-needle aspiration in the diagnosis and preoperative evaluation of pancreatic neuroendocrine tumors-experience with 76 cases. Gastrointest Endosc 2002;56:S118.

12. Baker MS, Knuth JL, DeWitt J. Pancreatic cystic neuroendocrine tumors: preoperative diagnosis with endoscopic ultrasound and fineneedle immunocytology. J Gastrointest Surg 2008;12:450-6.

13. Kongkam P, Al-Haddad M, Attasaranya S, O Neil J, Pais S, Sherman S, DeWitt J. EUS and clinical characteristics of cystic pancreatic neuroendocrine tumors. Endoscopy 2008;40:602-5.

14. Gornals J, Varas MJ, Catalá I, Maisterra S, Pons C, Bargalló D, et al Definitive diagnosis of neuroendocrine tumors using fine-needle aspiration-puncture guided by endoscopic ultrasonography. Rev Esp Enferm Dig 2011;103:123-8.

15. Suzuki H, Yamao K, Sawaki A, Mizuno N, Hara K, Hijoka S, et al. Clinical effectiveness of endoscopic ultrasound-guided fine-needle aspiration (EUS-FNA) for diagnosis of pancreatic neuroendocrine tumors (PNETs). GIE 2010;71 (5):AB222.

16. Carrara S, Arcidiacono PG, Mezzi G, Boemo C, Testoni PA. Contrastenhanced endoscopic ultrasound (CE-EUS) in the evaluation of pancreatic masses. JOP. J Páncreas (on line) 2006;7(Supl.5):558.

17. Dietrich Ch F, Ignee A, Braden B, Barreiros AP, Ott M, Hocke M Improved differentiation of pancreatic tumors using contrast-enhanced endoscopic ultrasound. Clin Gastroenterol Hepatol 2008;6:590-7.

18. Hirche TO, Ignee A, Barreiros AP, Schreiber-Dietrich D, Jungblut S, $\mathrm{Ott} \mathrm{M}$, et al. Indications and limitations of endoscopic ultrasound elastography for evaluation of focal pancreatic lesions. Endoscopy 2008;40:910-17.

19. Iglesias García J, Lariño-Noia J, Abdulkader I, Forteza J, DomínguezMuñoz JE. Quantitative endoscopic ultrasound elastography: an accurate method for the differentiation of solid pancreatic masses. Gastroenterology 2010;139:1172-80.

20. Fusaroli P, Spada A, Mancino MG, Caletti G. Contrast harmonic echoendoscopic ultrasound improves accuracy in diagnosis of solid pancreatic masses. Clin Gastroenterol Hepatol 2010;8:629-34.

21. Ishikawa T, Itoh A, Kawashima N, Ohno E, Matsubara H, Itoh Y, et al. Usefulness of EUS combined with contrast-enhancement in the differential diagnosis of malignant versus benign and preoperative localization of pancreatic endocrine tumors. Gastrointest Endosc 2010;71:951-9.

22. Varas Lorenzo M, Muñoz-Agel F, Cugat Andorra E, Gornals Soler J. Ultrasonographic contrast agents versus sonoelastography in digestive diseases. Rev Esp Enferm Dig 2011;103:204-8.

23. Rickes S, Unkrodt K, Ocran K, Neye H, Wermke W. Differentiation of neuroendocrine tumors from other pancreatic lesions by echo-enhanced power doppler sonography and somatostatin receptor scintigraphy. Pancreas 2003;26:76-81.

24. Gouya H, Vignaux O, Augui J, Dousset B, Palazzo L, Louvel A, et al $\mathrm{CT}$, endoscopic sonography, and combined protocol for preoperative evaluation of pancreatic insulinomas. AJR 2003;181:987-92.

25. Rockal AG, Reznek RH. Imaging of neuroendocrine tumours (CT/MR/US). Best Pract Res Clin Endocrinol Metab 2007;21:43-68.

26. Druce MR, Muthuppalaniappan VM, O Leary B, Drake W, Akker S, Rockall A, et al. Diagnosis and localisation of insulinoma: the value of modern MRI in conjunction with calcium stimulation catheterisation. Eur J Endocrinol 2010;162:971-8.

27. Gabriel M, Decristoforo C, Kendler D, Dobrozemsky G, Heute D, Uprimny C, et al. 68Ga-DOTA-Tyr3-octreotide PET in neuroendocrine 
tumors: comparison with somatostatin receptor scintigraphy and CT. J Nucl Med 2007;48:508-18.

28. Ambrosini V, Tomassetti P, Castellucci P, Campana D, Montini G, Rubello D, et al. Comparison between 68Ga-DOTA-NOC and 18FDOPA PET for the detection of gastro-entero-pancreatic and lung neuro-endocrine tumours. Eur J Nucl Med Mol Imaging 2008;35:1431-8.

29. Koopmans KP, Neels OC, Kema IP, Elsinga PH, Sluiter WJ, Vanghillewe K, et al. Improved staging of patients with carcinoid and islet cell tumors with 18F-dihydroxy-phenil-alanine and 11C-5-hydroxy-trytophan positron emission tomography. J Clin Oncol 2008;26:1489-95.

30. Frilling A, Sotiropoulos G, Radtke A, Malago M, Bockisch A, Kuehl $\mathrm{H}$, et al. The impact of $68 \mathrm{Ga}$-DOTATOC positron emission tomography/computed tomography on the multimodal management of patients with neuroendocrine tumors. Annals Surg 2010;252:850-6.

31. Krausz Y, Freedman N, Rubinstein R, Lavie E, Orevi M, et al. (68) Ga-DOTA-NOC PET/CT imaging of neuroendocrine tumors: comparison with (111)In-DTPA-Octreotide (OctreoScan-R). Mol Imaging Biol 2010;1-6.

32. Binderup T, Knigge U, Loft A, Mortensen J, Pfeifer A, Federspiel B, et al. Functional imaging of neuroendocrine tumors: A head-to-head comparison of somatostatin receptor scintigraphy, 123-I-MIBG scintigraphy, and 18-F-FDG PET. J Nucl Med 2010;51:704-12.

33. Versari A, Camellini L, Carlinfante G, Frasoldati A, Nicoli F, Grassi F, et al. Ga-68 DOTATOC PET, endoscopic ultrasonography, and multidetector $\mathrm{CT}$ in the diagnosis of duodenopancreatic neuroendocrine tumors: a single-centre retrospective study. Clin Nucl Med 2010;35:3218.

34. Khashab MA, Yong E, Lennon AM, Shin EJ, Amateu S, Hruban RH, et al. EUS is still superior to multidetector computerized tomography for detection of pancreatic neuroendocrine tumors. GIE 2011;73:6916.
35. Varas MJ, Ponseti JM, Alastruè A, Durán C, Llebaría C, Ballesta C, et al. Pancreatic endocrine tumors or apudomas. Rev Esp Enferm Dig 2011;103:184-90.

36. Modlin IM, Oberg K, Chung DC, Jensen RT, de Herder WW, Thakker $\mathrm{RV}$, et al. Gastroenteropancreatic neuroendocrine tumors. Lancet Oncol 2008;9:61-72.

37. Turuga KK, Kvols LK. Recent progress in the understanding, diagnosis, and treatment of gastroenteropancreatic neuroendocrine tumors. Cancer J Clin 2011;61:113-32.

38. Tan EH, Tan CH. Imaging of gastroenteropancreatic neuroendocrine tumors. World J Clin Oncol 2011;2:28-43.

39. Proye C, Malvauz P, Pattou F. Noninvasive imaging of insulinomas and gastrinomas with endoscopic ultrasonography and somatostatin receptor scintigraphy. Surgery 1998;124:1134-43.

40. Rindi G, Klöppel G, Alhman H, Caplin M, Couvelard A, de Herder W, et al. TNM staging of foregut (neuro) endocrine tumors: a consensus proposal including a grading system. Virchows Arch 2006;449:395-401.

41. Strosberg JR, Cheema A, Kvols LK. Stage I non functioning neuroendocrine tumors of the pancreas: surgery or surveillance? J Clin Oncol 2011;29(Supl. 4):A349.

42. Malagò R, D Onifrio M, Zamboni GA, Faccioli N, Falconi M, Boninsegna L, et al. Contrast-enhanced sonography of nonfunctioning pancreatic neuroendocrine tumors. AJR 2009;192:424-30.

43. An L, Li W, Yao K, Liu R, Lv F, Tang J, Zhang S. Assessment of contrast-enhanced ultrasonography in diagnosis and preoperative localization of insulinoma. Eur J Radiol 2011;80:675-80.

44. Haynes AB, Deshpande V, Ingkakul T, Vagefi PA, Szymonifka J, Thayer SP, et al. Implications of incidentally discovered, non-functioning pancreatic endocrine tumors. Arch Surg 2011;146:534-8.

45. Atiq M, Bhutani MS, Bektas M, Lee JE, Gong Y, et al. EUS-FNA for PNET: a tertiary cancer center experience. Dig Dis Sci 2011; 29: 3044-9. 